\title{
Latest Belle results on Tau decays
}

\author{
Y. $\operatorname{Jin}^{1^{*}}$, D. A. Epifanov ${ }^{2,3}$, H. Aihara ${ }^{1}$, on behalf of the Belle collaboration \\ 1 The University of Tokyo, Tokyo, Japan \\ 2 Budker Institute of Nuclear Physics, SB RAS, Novosibirsk, Russian Federation \\ 3 Novosibirsk State University, Novosibirsk, Russian Federation \\ * jin@hep.phys.s.u-tokyo.ac.jp
}

October 31, 2018

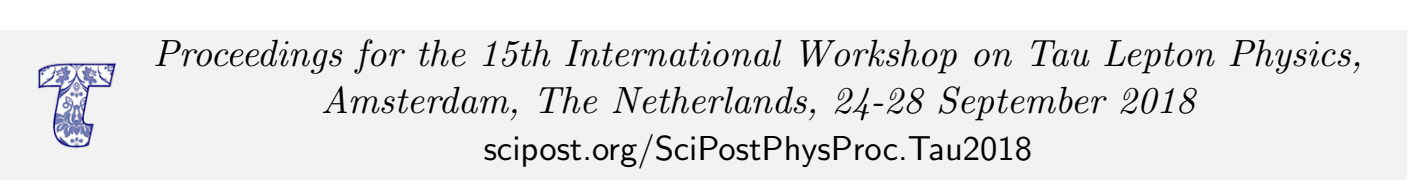

\begin{abstract}
We present preliminary results of a latest measurement at Belle, the branching fraction of rare tau decay, $\tau^{-} \rightarrow \pi^{-} \nu_{\tau} \ell^{+} \ell^{-}(\ell=e$ or $\mu)$, using a 562 $\mathrm{fb}^{-1}$ data set. The branching fraction of $\tau^{-} \rightarrow \pi^{-} e^{+} e^{-} \nu_{\tau}$ is measured to be $\mathcal{B}\left(\tau^{-} \rightarrow \pi^{-} e^{+} e^{-} \nu_{\tau}\right)=(2.11 \pm 0.19 \pm 0.30) \times 10^{-5}$, where the first uncertainty is statistical and the second is systematic. An upper limit is set on the branching fraction of $\tau^{-} \rightarrow \pi^{-} \mu^{+} \mu^{-} \nu_{\tau}, \mathcal{B}\left(\tau^{-} \rightarrow \pi^{-} \mu^{+} \mu^{-} \nu_{\tau}\right)<1.06 \times 10^{-5}$, at 90\% CL.
\end{abstract}

\section{Introduction}

The Belle detector, which was installed at the interaction point of the electron-positron asymmetric energy collider (KEKB), was working not only as a B-factory but also as a Tau-factory. The world's largest statistics of tau leptons was collected at Belle, which makes it possible to measure branching fractions for rare decays $\left(\mathcal{B} \sim 10^{-5}\right)$ of tau. We herein present the preliminary result of a latest measurement by Belle, the branching fraction of rare decay $\tau^{-} \rightarrow \pi^{-} \nu_{\tau} e^{+} e^{-}$. 1

A $\gamma^{*} W^{*} \pi$ vertex is involved in the $\tau^{-} \rightarrow \pi^{-} \ell^{+} \ell^{-} \nu_{\tau}$ decay, which plays an important role in calculating the radiative corrections to the $\tau^{-} \rightarrow \pi^{-} \nu_{\tau}$ decays [1] and in the evaluation of contributions from the hadronic light-by-light scattering to the muon anomalous magnetic moment [2]. The measured result of branching fraction could be used to validate the calculation [3] from Resonance Chiral Theory, a prevailing phenomenological approach to describe strong interaction at the energy region below tau mass. Meanwhile, rare decays of $\tau$ often serve as a probe of the physics beyond the Standard Model. A sterile neutrino could enter the diagrams of the decay mode of concern [4], resulting in observable enhancement on branching fraction. Also, a precise measurement of $\mathcal{B}\left(\tau^{-} \rightarrow \pi^{-} \nu_{\tau} \ell^{+} \ell^{-}\right)$ could facilitate searches for lepton flavour violating decays.

\section{Detector and MC simulations}

A $562 \mathrm{fb}^{-1}$ data set collected with Belle detector at the KEKB asymmetric-energy $\mathrm{e}^{+} \mathrm{e}^{-}$collider is exploited. The Belle detector is a general purpose large-solid-angle spec-

\footnotetext{
${ }^{1}$ unless otherwise specified, charge-conjugated decays are implied throughout the paper
} 
trometer consisting of a silicon vertex detector (SVD), a central drift chamber (CDC), an aerogel threshold Cherenkov counter (ACC), a barrel-like arrangement of time-of-flight scintillation counters (TOF), and an electromagnetic calorimeter (ECL) located inside a superconducting solenoid coil that provides a $1.5 \mathrm{~T}$ magnetic field. Outside the coil, an iron flux return yoke is instrumented to detect $K_{L}^{0}$ mesons and to identify muons (KLM). A detailed description of Belle detector can be found elsewhere 5 .

To determine the contamination of background processes and the detection efficiency of signal events, Monte Carlo (MC) samples are employed. The MC samples for hadronic processes are generated by EVTGEN package [6], KKMC [7] for fermion pairs, AAFH [8] for two-photon production of fermion pairs, and CTOY for cosmic ray muons. The decay of $\tau$ lepton is carried out by TAUOLA [9]. The final-state radiation of charged tracks is taken into account by using PHOTOS [10]. The response of the detector is simulated by a GEANT3-based program 11. Embedding the signal mode in TAUOLA, a signal generator is prepared according to Ref. [3]. The optimisation of selection criteria using MC samples is implemented by maximising a figure of merit defined as $S / \sqrt{S+B}$, where $S(B)$ stands for the number of signal (background) events.

\section{Event selection and reconstruction}

The selection is composed of two stages, aiming at suppressing background processes while retaining a high efficiency for the decay mode of concern. The first stage selects $e^{+} e^{-} \rightarrow \tau^{+} \tau^{-}$events and substantially reject the backgrounds from other processes that occur at $\Upsilon(4 S)$. The second stage proceeds to select one $\tau$ decay into $\pi^{-} \nu_{\tau} \ell^{+} \ell^{-}$, where a vertex fit is performed on the $\ell^{+} \ell^{-}$pair, and the other $\tau$ decay into one prong.

\subsection{Selection of $\tau^{+} \tau^{-}$events}

Events with four tracks and zero net charge are selected. The distance of the closest approach of each charged track to the interaction point must be within $\pm 5 \mathrm{~cm}$ along the beam direction (the $z$ axis) and $1 \mathrm{~cm}$ in the transverse plane (the $x-y$ plane). All four charged tracks are required to have transverse momentum larger than $0.1 \mathrm{GeV} / \mathrm{c}$ and the maximum of transverse momenta must exceed $0.5 \mathrm{GeV} / \mathrm{c}$. The total energy deposited in the ECL must be less than $10 \mathrm{GeV}$. A vertex fit is performed on all charged tracks to seek for the position of the primary vertex of the event, which is required to be near the interaction point within $0.5 \mathrm{~cm}$ in the $x-y$ plane and $\pm 2.5 \mathrm{~cm}$ along the $z$ axis. Photons, neutral tracks reconstructed from clusters in ECL, are selected with energy threshold 50 $\mathrm{MeV}$ in the barrel region $\left(32^{\circ}<\theta_{\gamma}<130^{\circ}\right)$ and $100 \mathrm{MeV}$ in the end cap region.

In the centre of mass (CMS) frame, the sum of the magnitudes of charged tracks' momenta is required to be less than $10 \mathrm{GeV} / \mathrm{c}$, meanwhile the sum of th magnitudes of charged tracks' momenta and neutral tracks' energies must be larger than $3 \mathrm{GeV}$. A missing momentum is defined as $P_{\text {miss }}=P_{\text {init }}-\sum_{i} P_{\text {trk }, i}-\sum_{i} P_{\gamma, i}$, where $P_{\text {init }}$ is the initial four momentum of the colliding $e^{+} e^{-}$beams, $\sum_{i} P_{\mathrm{trk}, i}$ and $\sum_{i} P_{\gamma, i}$ are the four momentum of the $i$ th charged and neutral tracks. Only events satisfying $1 \mathrm{GeV} / \mathrm{c}^{2}<M_{\text {miss }}<7 \mathrm{GeV} / \mathrm{c}^{2}$ and $30^{\circ}<\theta_{\text {miss }}<130^{\circ}$ are selected, where $M_{\text {miss }}$ and $\theta_{\text {miss }}$ are the mass and polar angle of the missing momentum in the CMS frame, respectively.

The event must satisfy at least one of the following additional conditions. 1, in the CMS frame, the sum of the magnitudes of charged tracks' momenta, neutral tracks' energies and the missing momentum must be less than $9 \mathrm{GeV}$. 2, in the CMS frame, the maximum of opening angles between two charged tracks must be larger than $175^{\circ} .3$, in the laboratory 
frame, at least two charged tracks are detected in the barrel region. 4, in the laboratory frame, the energy deposit of all charged tracks in ECL must be less than $5.3 \mathrm{GeV}$.

\subsection{Selection of $\tau^{-} \rightarrow \pi^{-} e^{+} e^{-} \nu_{\tau}$ events}

For each track, pion $\left(\mathcal{L}_{\pi}\right)$ and kaon $\left(\mathcal{L}_{K}\right)$ likelihoods are constructed from the ACC response, the specific ionization $(d E / d x)$ in the CDC and the flight-time measurement in the TOF. A likelihood ratio $\mathcal{P}_{K / \pi}=\mathcal{L}_{K} /\left(\mathcal{L}_{\pi}+\mathcal{L}_{K}\right)$ is formed and a cut $\mathcal{P}_{K / \pi}<0.6$ is applied to select pions. To select electrons, a likelihood ratio selection $\mathcal{P}_{e}=\mathcal{L}_{e} /\left(\mathcal{L}_{e}+\mathcal{L}_{x}\right)>$ 0.5 is applied, where the electron $\left(\mathcal{L}_{e}\right)$ and non-electron $\left(\mathcal{L}_{x}\right)$ likelihood functions include information on the specific ionization in the CDC $(d E / d x)$, the ratio of the energy of the cluster in the ECL to the momentum of the track, the transverse shape of the ECL shower and the signal amplitude in the ACC.

In the case of multiple $e^{+} e^{-}$vertex candidates, the one with the best fit quality is chosen. Pion candidates are required to have a momentum larger than $0.2 \mathrm{GeV} / \mathrm{c}$ both in the CMS and LAB frame and its $\mathcal{P}_{e}<0.2$. To recover bremsstrahlung effect, photon candidates that associate electron tracks with a cross angle within 0.05 radian and energy lower than that of electron in CMS frame are added to the electron track. Meanwhile, the invariant mass of new $e^{+} e^{-}$pair must be less than $0.05 \mathrm{GeV} / \mathrm{c}^{2}$. The fourth charged track is regarded as the daughter of the accompanying $\tau$.

To mitigate backgrounds due to external gamma conversion, cuts on the position of $e^{+} e^{-}$vertex are applied. Transverse position of $e^{+} e^{-}$vertex $\left(\left|R_{x y}\right|\right)$ must be less than $1.2 \mathrm{~cm}$, and longitudinal position of $e^{+} e^{-}$vertex $(Z)$ must be in the range of $[-1 \mathrm{~cm}$, $1.5 \mathrm{~cm}]$. To reduce hadronic background, thrust magnitude and the cosine of the angle between the momentum of the $\tau$ in signal hemisphere and the $\pi \ell \ell$ system in the CMS frame are employed. The thrust magnitude is defined as $\sum_{i}\left|\overrightarrow{p_{i}} \cdot \overrightarrow{n_{T}}\right| / \sum_{i}\left|\overrightarrow{p_{i}}\right|$, where $\mid \overrightarrow{p_{i}}$ is the momentum of the $i$ th particle in the CMS frame, either a charged or neutral track, and must locate in the range of $[0.85,0.99]$. The angle is calculated under the assumption that the massless neutrino is not reconstructed:

$$
\cos \theta_{\tau-3 \pi e e} \equiv \frac{2 E_{\mathrm{beam}} E_{\pi e e}-m_{\tau}^{2}-M_{\pi e e}^{2}}{2\left|P_{\tau}\right| \cdot\left|P_{\pi e e}\right|},
$$

where $E_{\text {beam }}$ is the energy of beam $(5.29 \mathrm{GeV})$, and $E_{\pi e e}, P_{\pi e e}$ and $M_{\pi e e}$ are the energy, momentum and invariant mass of the $\pi e e$ system, respectively. The $\cos \theta_{\tau-3 \pi e e}$ is required to locate in physical region $\left(\left|\cos \theta_{\tau-3 \pi \ell \ell}\right| \leq 1\right)$. To suppress $\tau^{-} \rightarrow \pi^{-} \pi^{0} \nu_{\tau}$, $\gamma$ veto and $\pi^{0}$ veto are employed. In the signal hemisphere, at most one photon is allowed with energy less than $300 \mathrm{MeV}$. A $\pi^{0}$ is reconstructed by two photons $\left(110<M_{\gamma \gamma}<160 \mathrm{MeV} / \mathrm{c}^{2}\right)$ or one photon and a pair of electron $\left(110<M_{\mathrm{e}^{+} \mathrm{e}^{-\gamma}}<165 \mathrm{MeV} / \mathrm{c}^{2}\right)$. Also, to further suppress the backgrounds from the $\rho^{-}\left(\pi^{-} \pi^{0}\right)$ resonance, the invariant mass of $\pi \ell \ell$ system $\left(M_{\pi \ell \ell}\right)$ is required to be larger than $1.05 \mathrm{GeV}$ and less than $1.8 \mathrm{GeV}$.

The detection efficiency of the $\tau^{-} \rightarrow \pi^{-} e^{+} e^{-} \nu_{\tau}$ events is found to be $(1.88 \pm 0.07) \%$. The main background comes from $\tau^{-} \rightarrow \pi^{-} \pi^{0} \nu_{\tau} \rightarrow \pi^{-} e^{+} e^{-} \gamma \nu_{\tau}$ (55.83\%) and $\tau^{-} \rightarrow$ $\pi^{-} \pi^{0} \nu_{\tau} \rightarrow \pi^{-} \gamma \gamma \nu_{\tau}(24.22 \%)$.

\subsection{Selection of $\tau^{-} \rightarrow \pi^{-} \mu^{+} \mu^{-} \nu_{\tau}$ events}

Charged tracks with $\mathcal{P}_{K / \pi}<0.6$ and $\mathcal{P}_{e}<0.8$ are selected as pion candidates. A likelihood ratio cut $\mathcal{P}_{\mu}=\mathcal{L}_{\mu} /\left(\mathcal{L}_{\mu}+\mathcal{L}_{\pi}+\mathcal{L}_{K}\right)>0.7$ is applied to select muons. The $\mathcal{L}_{\mu}$ and $\mathcal{L}_{x}(x=\pi, K)$ likelihood functions are calculated from two variables: the difference between the ranges calculated from the momentum of the particle and measured by KLM, and the $\chi^{2}$ of the KLM hits with respect to the track prolongated from CDC to KLM. 
In the case of multiple $\mu^{-} \mu^{+}$vertices, the candidate owning the smallest angle between $\mu^{-}$and $\mu^{+}$in the CMS frame is chosen. Only events with a $\mu^{-} \mu^{+}$vertex whose transverse radius $\left(R_{x y}\right)$ is less than $0.15 \mathrm{~cm}$ and invariant mass less than $0.85 \mathrm{GeV}$ are selected.

To reduce backgrounds from $\tau^{-} \rightarrow \pi^{-} \pi^{+} \pi^{-} \nu_{\tau}$ mode, a stringent cut $\mathcal{P}_{\mu}>0.97$ is imposed upon muon candidates and their transverse momentum are required larger than $720 \mathrm{MeV} / \mathrm{c}$ to ensure hitting the KLM detector. Meanwhile muon candidates are required to have $\mathcal{P}_{K / \pi}<0.8$ to suppress $\tau^{-} \rightarrow K^{-} \pi^{+} \pi^{-} \nu_{\tau}$ mode. To mitigate backgrounds from hadronic processes, the event is required to have a thrust magnitude larger than 0.9 in the CMS frame. The sum of energies of three final state charged tracks $(\pi, \mu, \mu)$ of signal $\tau$ must be less than the energy of beam. Also, pseudo mass of $\pi \mu \mu$ system is required to be less than $1.8 \mathrm{GeV} / \mathrm{c}^{2}$. The pseudo mass of $\pi \mu \mu$ system is defined as:

$$
m^{*}=\sqrt{2 \cdot E_{\pi \mu \mu}\left(E_{\text {beam }}-E_{\pi \mu \mu}\right)+M_{\pi \mu \mu}^{2}-2 \cdot\left|P_{\pi \mu \mu}\right|\left(E_{\text {beam }}-E_{\pi \mu \mu}\right)},
$$

where $E_{\pi \mu \mu}, P_{\pi \mu \mu}$ and $M_{\pi \mu \mu}$ are the energy, momentum and invariant mass of the $\pi \mu \mu$ system, respectively. To mitigate backgrounds involving $\pi^{0}, \gamma$ veto is employed. In the signal hemisphere, the sum of energies of all photons is required to be less than $300 \mathrm{MeV}$. Moreover, the number of photons in both hemispheres must be less than 6 .

The detection efficiency of the $\tau^{-} \rightarrow \pi^{-} \mu^{+} \mu^{-} \nu_{\tau}$ events is found to be $(4.13 \pm 0.16) \%$. The main background comes from $\tau^{-} \rightarrow \pi^{-} \pi^{+} \pi^{-} \nu_{\tau}$ (81.88\%) and $\tau^{-} \rightarrow \pi^{-} \pi^{+} \pi^{-} \pi^{0} \nu_{\tau}$ $(8.26 \%)$.

\section{Results}

\subsection{Determination of the branching fraction}

The signal yield is assumed to be the difference between the number of observed events and the number of background events predicted by the MC samples. The branching fraction of $\tau^{-} \rightarrow \pi^{-} \ell^{+} \ell^{-} \nu_{\tau}$ is calculated in adhering to the following formula:

$$
\mathcal{B}\left(\tau^{-} \rightarrow \pi^{-} \ell^{+} \ell^{-} \nu_{\tau}\right)=\frac{N_{\text {obs }}-N_{\mathrm{BKG}}}{\sigma_{\tau \tau} \cdot \mathcal{L} \cdot \epsilon_{\mathrm{sig}}},
$$

where $\sigma_{\tau \tau}=0.919 \pm 0.003 \mathrm{nb}[12]$ is the cross section of $\tau \tau$ production at $\Upsilon(4 S), \mathcal{L}$ the luminosity of exploited experimental data, $\epsilon_{\text {sig }}$ the detection efficiency of signal events, $N_{\text {obs }}$ the number of observed events from experimental data and $N_{\text {BKG }}$ the number of background events predicted by the MC.

\subsection{Estimation of systematic uncertainty}

For the measurement of branching fractions, the following sources of systematics are taken into account. The uncertainty due to the charged tracks' finding efficiency is estimated by using the partially reconstructed $D^{*-} \rightarrow D^{0} \pi^{-}, D^{0} \rightarrow \pi^{-} \pi^{+} K_{S}^{0}$ events. The uncertainty due to particle identification (PID) includes the contribution from the $\mathcal{P}_{K / \pi}$ requirement, which is investigated by a control sample of $D^{*+} \rightarrow D^{0} \pi_{\text {slow }}^{+}, D^{0} \rightarrow K^{-} \pi^{+}$, and the contribution from lepton identification studied by the $\gamma \gamma \rightarrow \ell^{+} \ell^{-}$and $J / \psi \rightarrow \ell^{+} \ell^{-}$ processes. In addition, the $\pi \rightarrow \mu$ mis-identification ( $\pi$ fake rate) is determined by a control sample of $\tau^{ \pm} \rightarrow \pi^{ \pm} \pi^{-} \pi^{+} \nu_{\tau}$. The uncertainty associated with luminosity is obtained by using Bhabha events where the final state electrons are required to be detected in the barrel region. The uncertainty associated with the trigger efficiency is investigated by a dedicated MC program. The uncertainty due to the $\pi^{0}$ reconstruction is investigated by 
a control sample of $\tau^{-} \rightarrow \pi^{-} \pi^{0} \nu_{\tau}, \pi^{0} \rightarrow e^{+} e^{-} \gamma$. The uncertainty arising from the limited size of MC samples for the study of background contamination and efficiency of signal events is estimated via its binomial variation. The uncertainty of the branching fractions of background modes are also taken into account.

\section{$4.3 \mathcal{B}\left(\tau^{-} \rightarrow \pi^{-} e^{+} e^{-} \nu_{\tau}\right)$}

After all selection criteria, 676 events are observed in the signal box of $\tau^{-} \rightarrow \pi^{-} \nu_{\tau} e^{+} e^{-}$ with a background expectation of $478 \pm 23$ events and 689 events are observed in the signal box of the charge-conjugate mode, $\tau^{+} \rightarrow \pi^{+} \bar{\nu}_{\tau} e^{+} e^{-}$, with a background expectation of 476 \pm 22 events, as shown in Figure 1, where daggers over-number the colourful histogram. A clear excess of events above the expected background is observed, which gives rise to a branching fraction $\mathcal{B}\left(\tau^{-} \rightarrow \pi^{-} e^{+} e^{-} \nu_{\tau}\right)=(2.11 \pm 0.19 \pm 0.30) \times 10^{-5}$, where the first uncertainty is statistical and the second one is systematic. The systematic uncertainties are summarized in Table 1,

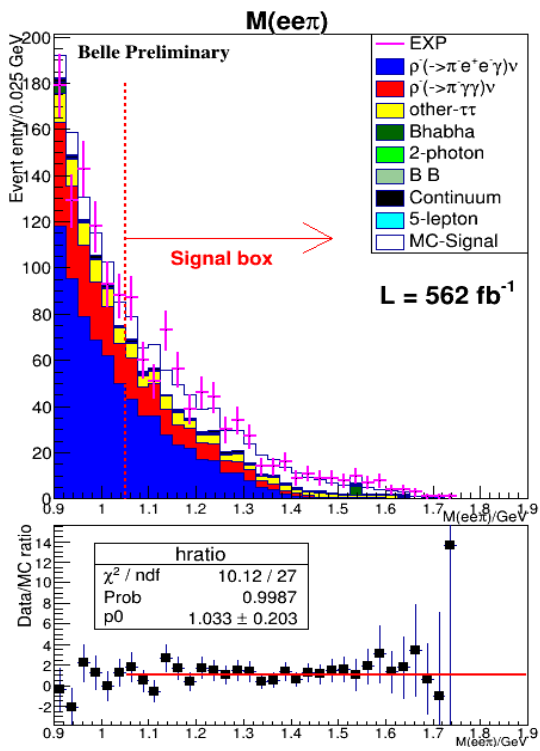

(a) Signal box of $\tau^{-} \rightarrow \pi^{-} \nu_{\tau} e^{+} e^{-}$.

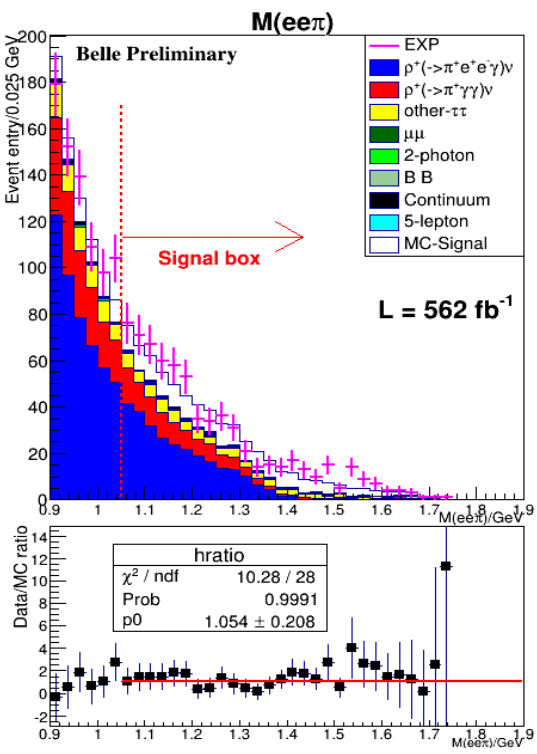

(b) Signal box of $\tau^{+} \rightarrow \pi^{+} \nu_{\tau} e^{+} e^{-}$.

Figure 1: Distribution of the invariant mass of $e^{+} e^{-} \pi(M(e e \pi))$. Daggers stand for the observed data and histogram for MC. MC sample is normalized with respect to the luminosity.

Table 1: The breakdown of systematic uncertainties.

\begin{tabular}{|c|c|}
\hline Luminosity & $4.66 \%$ \\
Tracking & $4.66 \%$ \\
PID & $11.14 \%$ \\
MC size & $3.74 \%$ \\
$\tau \tau$ cross section & $0.3 \%$ \\
Trigger & $1.16 \%$ \\
$\pi^{0}$ veto & $1.86 \%$ \\
$\mathcal{B}$ 's of BKG modes & $4.42 \%$ \\
\hline In total & $14.4 \%$ \\
\hline
\end{tabular}




\section{$4.4 \mathcal{B}\left(\tau^{-} \rightarrow \pi^{-} \mu^{+} \mu^{-} \nu_{\tau}\right)$}

After all selection criteria, 1315 events are observed in the signal box of $\tau^{-} \rightarrow \pi^{-} \nu_{\tau} \mu^{+} \mu^{-}$ with a background expectation of $1129 \pm 55$ events and 1263 events are observed in the signal box of the charge-conjugate mode, $\tau^{+} \rightarrow \pi^{+} \bar{\nu}_{\tau} \mu^{+} \mu^{-}$, with a background expectation of $1115 \pm 54$ events, as shown in Figure 2. The observed events are consistent with the background expectation within $3 \sigma$. The systematic uncertainties of background expectation includes luminosity, tracking, particle identification, MC sizes, trigger, accuracies of branching fractions of background modes and $\pi \rightarrow \mu$ mis-identification calibration. As a result, an upper limit is set on this decay mode taking into account the systematic uncertainty of background expectation and the statistical uncertainty of observed events, $\mathcal{B}\left(\tau^{-} \rightarrow \pi^{-} \mu^{+} \mu^{-} \nu_{\tau}\right)<1.06 \times 10^{-5}$ at $90 \% \mathrm{CL}$.

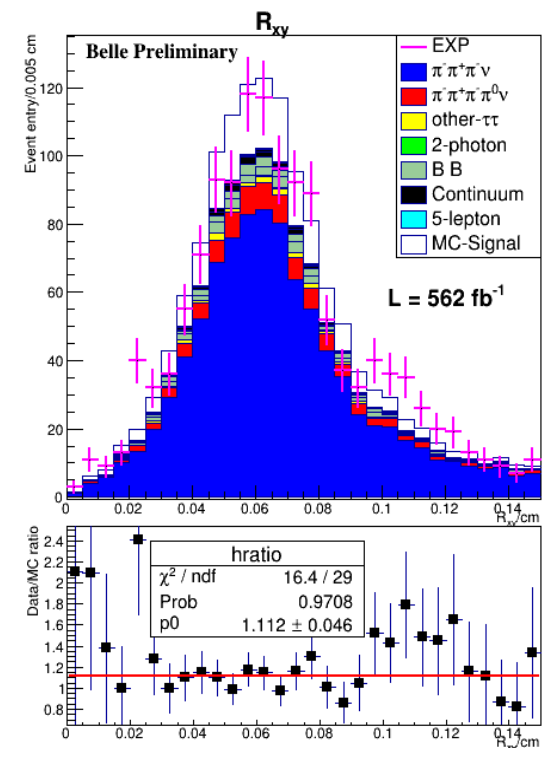

(a) Signal box of $\tau^{-} \rightarrow \pi^{-} \nu_{\tau} \mu^{+} \mu^{-}$

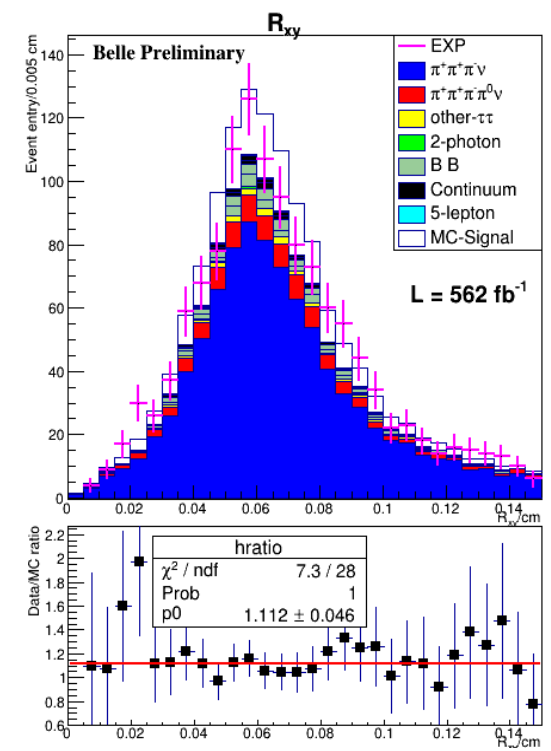

(b) Signal box of $\tau^{+} \rightarrow \pi^{+} \nu_{\tau} \mu^{+} \mu^{-}$.

Figure 2: Distribution of the transverse distance of $\mu^{+} \mu^{-}$vertex $\left(\mathrm{R}_{\mathrm{xy}}\right)$. Daggers stand for the observed data and histogram for MC. MC sample is normalized with respect to the luminosity.

\section{Conclusion}

Using a $562 \mathrm{fb}^{-1}$ data set collected at the $\Upsilon(4 S)$ resonance with Belle detector at the KEKB asymmetric-energy $e^{+} e^{-}$collider, the branching fraction of $\tau^{-} \rightarrow \pi^{-} e^{+} e^{-} \nu_{\tau}$ is obtained for the first time, $\mathcal{B}\left(\tau^{-} \rightarrow \pi^{-} e^{+} e^{-} \nu_{\tau}\right)=(2.33 \pm 0.19 \pm 0.30) \times 10^{-5}$, where the first error is statistical and the second error is systematic, with $5.9 \sigma$ significance to reject background-only hypothesis. An upper limit is set on the branching fraction of $\tau^{-} \rightarrow \pi^{-} \mu^{+} \mu^{-} \nu_{\tau}, \mathcal{B}\left(\tau^{-} \rightarrow \pi^{-} \mu^{+} \mu^{-} \nu_{\tau}\right)<1.06 \times 10^{-5}$, at $90 \% \mathrm{CL}$. The measured result is consistent with the theoretical prediction from the Standard Model and could facilitate searches for physics beyond the Standard Model. 


\section{A MC samples for $\tau^{-} \rightarrow \pi^{-} \pi^{0} \nu_{\tau}$}

It is worth mentioning that the MC samples of $\tau^{-} \rightarrow \pi^{-} \pi^{0} \nu_{\tau}$, main background in the analysis of $\tau^{-} \rightarrow \pi^{-} e^{+} e^{-} \nu_{\tau}$, is generated according to the Belle pion form factor 13. The pion form factor obtained by Belle is consisted with the previous CLEO result at the peak region while differ in the high mass region as shown in Figure 3 . In this analysis, the pion form factor from Belle is adopted due to its higher precision.

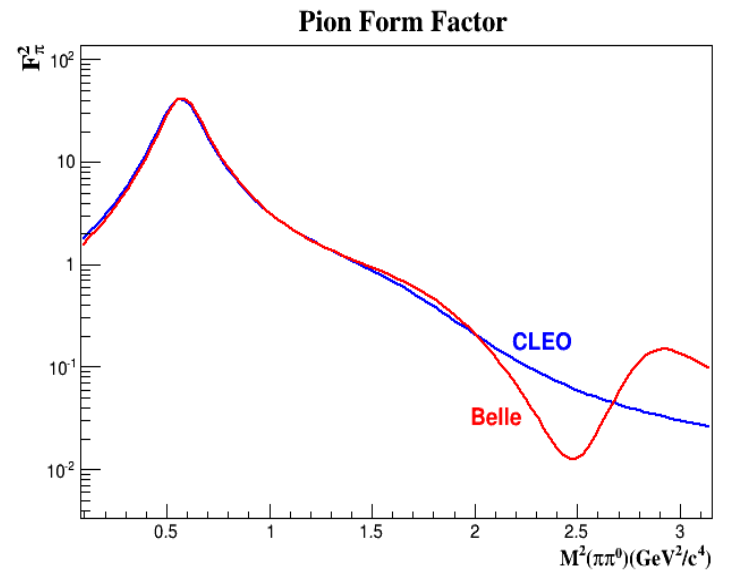

Figure 3: Comparison of pion form factor obtained by Belle to that of CLEO.

\section{References}

[1] R. Decker, M. Finkemeier, Radiative corrections to the decay $\tau \rightarrow \pi(K) \nu_{\tau}$, Physics Letters B, 334, 199 (1994) doi:10.1016/0370-2693(93)90345-I

[2] J. P. Miller et al., Muon(g-2): experiment and theory, Reports on Progress in Physics, 70, 795 (2007) doi:10.1088/0034-4885/70/5/R03

[3] P. Roig, et al., Weak radiative pion vertex in $\tau^{-} \rightarrow \pi^{-} \nu_{\tau} l^{+} l^{-}$decays, Physical Review D, 88, 033007 (2013) doi:10.1103/PhysRevD.88.033007

[4] C. Dib, et al., Heavy sterile neutrinos in tau decays and the MiniBooNE anomaly, Physical Review D, 85, 011301 (2012), doi:10.1103/PhysRevD.85.011301

[5] A. Abashian et al., The Belle detector, Nuclear Instruments and Methods in Physics Research Section A 479, 117 (2002), doi:10.1016/S0168-9002(01)02013-7.

[6] D. J. Lange, The EvtGen particle decay simulation package, Nuclear Instruments and Methods in Physics Research Section A 462, 152 (2001), doi:10.1016/S01689002(01)00089-4

[7] S. Jadach, B. Ward, Z. Was, The precision Monte Carlo event generator KK for twofermion final states in $e^{+} e^{-}$collisions, Computer Physics Communications, 130, 260 (2000), doi:10.1016/S0010-4655(00)00048-5

[8] F. A. Berends, P. H. Daverveldt, R. Kleiss, Monte Carlo simulation of two-photon processes: II: Complete lowest order calculations for four-lepton production processes 
in electron-positron collisions, Computer Physics Communications, 40, 285 (1986), doi: $10.1016 / 0010-4655(86) 90115-3$

[9] N. Davidson, et al., Universal interface of TAUOLA: technical and physics documentation, Computer Physics Communications, 183, 821 (2012), doi:10.1016/j.cpc.2011.12.009

[10] E. Barberio, B. van Eijk, and Z. Was, Photos - a universal Monte Carlo for QED radiative corrections in decays, Computer Physics Communications, 66, 115 (1991), doi:10.1016/0010-4655(91)90012-A

[11] R. Brun et al., GEANT 3.21, CERN Report No.DD/EE/84-1, 1987.

[12] S. Banerjee, et al., Tau and muon pair production cross sections in electronpositron annihilations at $\sqrt{s}=10.58 \mathrm{GeV}$, Physical Review D, 77, 054012 (2008), doi:10.1103/PhysRevD.77.054012

[13] M. Fujikawa, H. Hayashii, S. Eidelman, et al., High-statistic study of the $\tau^{-} \rightarrow \pi^{-} \pi^{0} \nu_{\tau}$ decay, Physical Review D, 78072006 (2008) doi:10.1103/PhysRevD.78.072006 